Research Article

\title{
Real life clinical management and survival in cutaneous malignant melanoma: the Italian Clinical National Melanoma Registry (CNMR) experience
}

Anna Crispo ${ }^{1}$, Maria Teresa Corradin ${ }^{2}$, Erika Giulioni², Antonella Vecchiato ${ }^{3}$, Paolo Del Fiore $^{3}$, Paola Queirolo ${ }^{4-5}$, Francesco Spagnolo ${ }^{4}$, Vito Vanella ${ }^{1}$, Corrado Caracò ${ }^{1}$, Giulio Tosti ${ }^{5}$, Elisabetta Pennacchioli ${ }^{5}$, Giuseppe Giudice ${ }^{6}$, Eleonora Nacchiero ${ }^{6}$, Pietro Quaglino ${ }^{7}$, Simone Ribero $^{7}$, Monica Giordano ${ }^{8}$, Desire Marussi ${ }^{8}$, Stefania Barruscotti ${ }^{9}$, Michele Guida ${ }^{10}$, Vincenzo De Giorgi ${ }^{11}$, Marcella Occelli ${ }^{12}$, Federica Grosso ${ }^{13}$, Giuseppe Cairo ${ }^{14}$, Alessandro Gatti $^{15}$, Daniela Massa ${ }^{16}$, Laura Atzori ${ }^{17}$, Nicola Calvani ${ }^{18}$, Tommaso Fabrizio ${ }^{19}$, Giuseppe Mastrangelo ${ }^{20}$, Federica Toffolutti ${ }^{21}$, Egidio Celentano ${ }^{1}$, Mario Budroni ${ }^{22}$, Sara Gandini ${ }^{5}$, Carlo Riccardo Rossi ${ }^{3-20}$, Alessandro Testori ${ }^{9}$,Giuseppe Palmieri ${ }^{23}$, and Paolo A. Ascierto ${ }^{1^{*}}$ for the ${ }^{\wedge}$ Clinical National Melanoma Registry Study Group at the Italian Melanoma Intergroup

Affiliations:

1. Istituto Nazionale Tumori IRCCS Fondazione G. Pascale, Napoli, Italy

2. Presidio Ospedaliero di Pordenone, Italy

3. Istituto Oncologico Veneto IOV - IRCCS, Padova, Italy

4. IRCCS Ospedale Policlinico San Martino, Genova, Italy

5. Istituto Europeo di Oncologia - IRCCS, Milano, Italy

6. Università degli Studi di Bari Aldo Moro, Bari, Italy

7. Università degli Studi di Torino, Torino, Italy

8. Ospedale Sant'Anna, Como, Italy

9. Fondazione I.R.C.C.S. Policlinico San Matteo, Pavia, Italy

10. IRCCS Istituto Tumori "Giovanni Paolo II", Bari, Italy

11. Università degli Studi di Firenze, Firenze, Italy

12. A.O. Santa Croce e Carle, Cuneo, Italy

13. Azienda Ospedaliera SS Antonio e Biagio e Cesare Arrigo, Alessandria, Italy

14. Presidio Ospedaliero "Vito Fazzi", Lecce, Italy

15. ULSS 2 Marca Trevigiana Ospedale Ca' Foncello Treviso, Italy

16. Ospedale Businco, Cagliari, Italy

17. Università degli Studi di Cagliari, Italy

18. Stabilimento Ospedaliero "A. PERRINO", Brindisi, Italy

19. IRCCS Centro di Riferimento Oncologico Basilicata, Rionero in Vulture, Italy

20. Università degli Studi di Padova, Padova, Italy

21. Centro di Riferimento Oncologico di Aviano (CRO) IRCCS, Italy

22. Registro Tumori Provincia di Sassari, Azienda Ospedaliera Universitaria Sassari, Italy

23. Istituto di Ricerca Genetica e Biomedica, CNR, Sassari, Italy

^ Clinical National Melanoma Registry Study Group at the Italian Melanoma Intergroup: ASEROSALVATORE Azienda Ospedaliera di Rilievo Nazionale e di Alta Specializzazione Garibaldi-Nesima 
BARBATI ROSANNA ASL Roma C-Ospedale S.Eugenio BIANCHI LUCA Azienda Ospedaliera Universitaria Policlinico Tor Vergata BRUDER FRANCESCA Ospedale Oncologico CATRICALÀ CATERINA Istituto Dermatologico San Gallicano IRCCS - IFO CINIERI SAVERIO Presidio Ospedaliero Antonio Perrino DEL VECCHIO MICHELE Fondazione I.R.C.C.S. Istituto Nazionale dei Tumori DI FILIPPO FRANCO Istituto Nazionale Tumori Regina Elena IRCCS - IFO FARGNOLI MARIA CONCETTA Presidio Ospedaliero San Salvatore FERRELI CATERINA Università degli Studi di Cagliari-Azienda Ospedaliero Universitaria FIERRO MARIA TERESA A.O.U. Città della Salute e della Scienza - P.O. San Lazzaro FORCIGNANÒ ROSACHIARA Azienda Ospedaliera Vito Fazzi GUIDOBONI MASSIMO I.R.S.T. Istituto Scientifico Romagnolo per lo Studio e la Cura dei Tumori IACONO CARMELO Azienda Ospedaliera Sanitaria 7 Ragusa-Ospedale Maria Paternò Arezzo LOSPALLUTI LUCIA Azienda Sanitaria Locale BA-Ospedale di Venere MAIO MICHELE Azienda Ospedaliera Universitaria Senese Ospedale Le Scotte MASCHERONI LUIGI Humanitas - Casa di Cura San Pio X s.r.l.

MILESI LAURA Azienda Ospedaliera Papa Giovanni XXIII MOISE GIANMICHELE Azienda per i Servizi Sanitari $n \circ 2$ Isontina Ospedale di Gorizia MORETTI GIOVANNA Azienda Ospedaliera Ospedali Riuniti Papardo-Piemonte PELLICANO RICCARDO IRCCS Casa Sollievo della Sofferenza PIZZICHETTA MARIA ANTONIETTA Centro di Riferimento Oncologico, Istituto Nazionale Tumori RINALDI GAETANA Azienda Ospedaliera Universitaria Policlinico 'Paolo Giaccone’ SANNA GIOVANNI Azienda Ospedaliero-Universitaria di Sassari STAIBANO STEFANIA Azienda Ospedaliera Universitaria Federico II di Napoli STANGANELLI IGNAZIO Ospedale S. Maria delle Croci - USL di Ravenna TREVISAN GIUSTO Ospedale Maggiore - Azienda Ospedaliera Universitaria di Trieste VISINI MARILENA A.O. di Lecco Presidio Ospedaliero Alessandro Manzoni ZANNETTI GUIDO Azienda Ospedaliero-Universitaria di Bologna Policlinico S. Orsola-Malpighi ZICHICHI LEONARDOAzienda Sanitaria Provinciale - Presidio Ospedaliero di Trapani

\section{${ }^{*}$ Correspondence:}

Paolo A. Ascierto, MD

Melanoma, Cancer Immunotherapy and Development Therapeutics Unit

Istituto Nazionale Tumori IRCCS Fondazione "G. Pascale"

Via Mariano Semmola

80131 Napoli - Italy

Tel +390815903236

Fax +390815903841

email: paolo.ascierto@gmail.com; p.ascierto@istitutotumori.na.it 


\section{Simple Summary:}

Cutaneous malignant melanoma (CMM) is one of the most aggressive types of skin cancer. The aim of this study is to investigate the oncological treatments employed in the real-life clinical management of patients with advanced CMM in several Italian centers which are part of the Clinical National Melanoma Registry (CNMR. The immunotherapy can be considered the best therapy to improve survival in a real-world-population of cutaneous malignant melanoma. In the future, the main challenge of CNMR is the identification of the best therapy that patients could benefit to improve survival.

\section{Abstract:}

Background: Cutaneous malignant melanoma (CMM) is one of the most aggressive types of skin cancer. Currently, innovative approaches such as target therapies and immunotherapies have been introduced in clinical practice for the treatment of metastatic CMM. Data of clinical trials and real life studies that evaluate the outcomes of these therapeutic associations are necessary to establish their clinical utility. The aim of this study is to investigate the types of oncological treatments employed in the real-life clinical management of patients with advanced CMM in several Italian centers which are part of the Clinical National Melanoma Registry (CNMR), and the oncological outcomes obtained.

Methods: CNMR collects data of patients with a histologically confirmed diagnosis of primary CMM treated in one of the 38 Italian institutions (hospitals, research institutes, etc.) participating in the network. Melanoma-specific survival and Overall survival were calculated. Kaplan-Meier curves and medians of OS and 95\% CI are presented overall and by immunotherapy and target treatments. The Log-rank test compared curves by treatments. Multivariate Cox regression models were used to estimate the hazard ratios adjusting for confounders and other prognostic factors.

Results: The median follow-up time was 36 months (range 1.2-185.1). 787 CMM were included in the analysis with completed information about therapies.Global immunotherapy showed a significant improved survival compared with all other therapies 
( $p=0.001$ ). $75 \%$ was the highest reduction of death reached by nivolumab/pembrolizumab immunotherapy (anti-PD1 HR=0.25 95\% CI 0.14-0.42), globally immunotherapy was significantly associated with improved survival, either for anti-CTL A4 monotherapy or combined with anti-PD1 (HR=0.47;95\% CI $0.33-0.66$ and $\mathrm{HR}=0.26$; 95\% CI $0.15-0.46$, respectively).

Conclusions: The nivolumab/pembrolizumab and the combination of ipilimumab can be considered the best therapy to improve survival in a real-world-population. The CNMR can complement clinical registries with the intent of improving cancer management and standardizing cancer treatment.

Keywords: medical record systems, cutaneous malignant melanoma, survival analysis, immunotherapy

\section{Graphical Abstract}

\begin{tabular}{|c|c|c|c|}
\hline \multirow[t]{2}{*}{ Parameter/Category } & \multicolumn{3}{|c|}{ Adjusted Multivariate Analysis‡ } \\
\hline & $\mathrm{HR}$ & $95 \% \mathrm{CI}$ & P \\
\hline \multicolumn{4}{|l|}{ Immunotherapy } \\
\hline No Bmmuno and No Target therapy & $1.0^{+}$ & & \\
\hline ANTI-PD 1 (Nitobumab/Pebrolizionab) & 0.25 & $0.147-0.43$ & $<0.0001$ \\
\hline ANTI-CTL A4 (Ipilimumab) & 0.47 & $0.33-0.67$ & $<0.0001$ \\
\hline ANTI-PD 1-ANTI-CTL A4 & 0.26 & $0.15-0.47$ & $<0.0001$ \\
\hline
\end{tabular}




\section{Introduction}

Cutaneous malignant melanoma (CMM) is one of the most aggressive types of skin cancer. The incidence of CMM has increased in Europe over the last years, and cohort studies suggest that the increasing trend of incidence will continue for at least the next 2 decades [1-3] Mortality rates have also increased in the last decades, especially in men, despite a clear decrease of Breslow tumor thickness in the USA and Europe [1,4]. In the USA, the raw mortality rates per 100,000 inhabitants per year increased from 2.8 to 3.1, with an estimate of 10,130 deaths from melanoma in 2016 (they were 8650 in 2009) [1]. In Italy, 12,3000 new cases and over 2,000 deaths were estimated in 2019 [5-6].

Surgery is currently the golden standard for patients with early stage CMM, who represent only part of the global cases. The treatment of patients with advanced stage CMM is more complex, as for decades no chemotherapy regimens have been found effective in prolonging survival. Currently, innovative approaches such as target therapies and immunotherapies have been introduced in clinical practice for the treatment of metastatic CMM. Target therapies are based on the use of drugs targeting specific genetic alterations in candidate genes, blocking specific pathways implicated in the oncogenesis of melanoma [7]. BRAF mutations represent currently the main molecular targets for melanoma treatment, as they involve approximately $50 \%$ of the cases, and identify patients who may benefit from treatment with BRAF inhibitors, like vemurafenib or dabrafenib [8-10]. Recently, the combination of anti-BRAF drugs with MEK inhibitors showed improved oncological outcomes in comparison to monotherapies (70\% one-year and 50\% two-years survival), with a better safety profile [11-13].

Immuno-therapy takes advantage of the fact that treatments indirectly affect cancer cells by stimulating the patient's immune system, particularly enhancing the immune system's T-cell response [14]. Ipilimumab, a monoclonal antibody that blocks the activity of the CTLA-4, has shown a long-term survival in about $20 \%$ of the patients treated [15-17]. Programmed death 1 (PD1) is a membrane receptor of tumor cells (its main ligand is PD-L1) that represents a powerful brake to the immune system's response, and the target of 
specific inhibitors (nivolumab and pembrolizumab) which have been recently introduced into clinical practice, as they were shown more effective than ipilimumab in terms of overall survival (OS) and toxicity [18-19]. Recent studies showed that the combination of anti-CTLA-4 and anti-PD-1 is more effective than monotherapy, but a higher incidence of high-grade adverse events was found [20]. Combinations of targeted therapies and immunotherapies are currently investigated; the advantage of such combinations is that more than one anti-tumoral mechanism are employed against CMM. Data of clinical trials and real life studies that evaluate the outcomes of these therapeutic associations are necessary to establish their clinical utility.

The aim of this study is to investigate the types of oncological treatments employed in the real-life clinical management of patients with advanced CMM in several Italian centers which are part of the Clinical National Melanoma Registry (CNMR), and the oncological outcomes obtained.

\section{Results}

A total of $8,163 \mathrm{CMM}$ cases were registered in CNMR in the period under investigation. $17 \%(1,401)$ were melanoma in situ, $63 \%$ (5121 patients) had a stage from IA to IIC, $20 \%$ had a stage from IIIA (Table 1).

Table 1. Distribution of melanoma patients by stage in the Italian Clinical National Melanoma Registry (CNMR), 2011-2017

\begin{tabular}{|c|c|c|}
\hline Melanoma Stage & N (\%) & $\%$ \\
\hline In situ & $1401(17 \%)$ & 17 \\
\hline IA & $2061(25 \%)$ & \\
\hline IB & $1444(18 \%)$ & \\
\hline IIA & $842(10 \%)$ & 63 \\
\hline IIB & $488(6 \%)$ & \\
\hline IIC & $286(4 \%)$ & \\
\hline IIIA & $229(3 \%)$ & \\
\hline IIIB & $275(3 \%)$ & \\
\hline IIIC & $247(3 \%)$ & 20 \\
\hline IV & 890 (11\%) & \\
\hline Total & 8163 & 100 \\
\hline
\end{tabular}


Table 2 shows the distribution of the melanoma stage in accordance to their demographic data, geographic origin: 4,242 (52\%) were males and the mean age was 58 $\pm 15 y e a r s, 3,916$ ( $48 \%$ ) were females and the mean age was $54 \pm 16$ years, the majority of the sample coming from northern Italian centers. Stage III counts 751 patients, but only 14\% were IIIB-IIIC unresectable that were included in the analysis.

Table 2. Socio-demographic characteristics according to Stage in the Italian Clinical National Melanoma Registry (CNMR), 2011-2017

STAGE

\begin{tabular}{|c|c|c|c|c|c|c|c|c|c|}
\hline & \multicolumn{2}{|c|}{ In situ } & \multicolumn{2}{|c|}{ I-II } & \multicolumn{2}{|c|}{ III $(A, B, C)$} & \multicolumn{2}{|c|}{ IV } & \multirow[b]{2}{*}{$\begin{array}{l}\text { Total } \\
8163 \\
\end{array}$} \\
\hline & $\begin{array}{c}N \\
1401\end{array}$ & $\begin{array}{l}(\%) \\
(17.1)\end{array}$ & $\begin{array}{c}N \\
5121\end{array}$ & $\begin{array}{c}(\%) \\
(62.7)\end{array}$ & $\begin{array}{c}\mathrm{N} \\
751\end{array}$ & $\begin{array}{l}(\%) \\
(9.3)\end{array}$ & $\begin{array}{c}N \\
890\end{array}$ & $\begin{array}{c}(\%) \\
(10.9)\end{array}$ & \\
\hline \multicolumn{10}{|l|}{ Gender } \\
\hline Male & 677 & $(48)$ & 2600 & (51) & 435 & (58) & 530 & $(60)$ & 4242 \\
\hline Female & 724 & (52) & 2521 & (49) & 315 & (41) & 356 & $(40)$ & 3916 \\
\hline missing & & & & & 1 & & 4 & & 5 \\
\hline \multicolumn{10}{|l|}{ Age } \\
\hline$\leq 40$ & 245 & (18) & 1005 & (20) & 105 & (14) & 90 & (10) & 1445 \\
\hline $41-50$ & 285 & $(20)$ & 1134 & (22) & 142 & (19) & 128 & (14) & 1689 \\
\hline $51-60$ & 253 & (18) & 1017 & (20) & 156 & (21) & 174 & (20) & 1600 \\
\hline $61-70$ & 310 & (22) & 1005 & (20) & 174 & (23) & 214 & (24) & 1703 \\
\hline$>70$ & 308 & (22) & 960 & (18) & 174 & (23) & 284 & (32) & 1726 \\
\hline \multicolumn{10}{|l|}{ Geographical area } \\
\hline North & 800 & (57) & 2584 & (50) & 550 & (73) & 590 & (66) & 4524 \\
\hline Center-South & 601 & (43) & 2537 & (50) & 201 & (27) & 300 & (34) & 3639 \\
\hline
\end{tabular}

Patients characteristics, data on tumor and BRAF mutational status were restricted to advanced melanoma (IIIB-IIIC unresectable, IV), and the total sample counted 787 patients. Regarding to stage $12 \%$ had aninitial diagnosis of in situ, $38 \%$ had an early diagnosis (IA-IIC), 37\% stage III and 13\% had a confirmed advanced melanoma stage (IV). $76 \%$ was the percentage of BRAF executed in our sample and the incidence of BRAF mutations was slightly greater than 50\%; most cases were analyzed after the year 2013 when target 
therapies were diffusely employed in clinical practice; in addition, more cases among those analyzed harbored stage IV tumors rather than stage IIIB-IIIC melanomas. (Table3)

Table.3 Tumor characteristics for Advanced Stage (IIIB-IIIC unresectable , IV)

\section{ADVANCED STAGE}

IIIB-IIIC (unresectable), IV

$\mathrm{N}=787$

\begin{tabular}{|c|c|}
\hline \multicolumn{2}{|l|}{ Gender } \\
\hline Male & $476(61)$ \\
\hline Female & 307 (39) \\
\hline \multicolumn{2}{|l|}{ Age } \\
\hline$\leq 60$ yrs & $355(45)$ \\
\hline$>60$ yrs & $432(55)$ \\
\hline \multicolumn{2}{|l|}{ BMI } \\
\hline$<25$ & $315(40)$ \\
\hline$\geq 25$ & $386(49)$ \\
\hline missing & $86(11)$ \\
\hline \multicolumn{2}{|l|}{ LDH } \\
\hline Normal & $479(61)$ \\
\hline Abnormal & $43(5)$ \\
\hline Unknown & $265(34)$ \\
\hline \multicolumn{2}{|l|}{ Initial Stage } \\
\hline In situ & $98(12)$ \\
\hline Stage I-II & $297(38)$ \\
\hline Stage III & $291(37)$ \\
\hline Stage IV & $101(13)$ \\
\hline \multicolumn{2}{|c|}{ BRAF executed } \\
\hline No & $120(15)$ \\
\hline Yes & $594(76)$ \\
\hline Not applicable & $73(9)$ \\
\hline \multicolumn{2}{|c|}{ Mutational status } \\
\hline Mutant & $322(54)$ \\
\hline Wild Type & $269(45.5)$ \\
\hline unknown & $3(0.5)$ \\
\hline \multicolumn{2}{|c|}{ Year BRAF executed } \\
\hline$<2013$ & $498(63)$ \\
\hline$\geq 2013$ & $289(37)$ \\
\hline
\end{tabular}

${ }^{*} 4$ patients did not report the gender 
The median follow-up time was 36 months (range 1.2-185.1). Observed patients and percentage according to type of treatment were reported in Table 4; total death events (for all causes and deaths for the diseases) were reported and median Melanoma-specific survival (M-s S) was calculated. As first line of treatment (choice), $41 \%$ of patients $(n=319)$ received immunotherapy, 36\% received BRAF-targeted therapies $(n=285), 35 \%$ received chemotherapy $(n=275), 35 \%$ received local therapy (electrochemotherapy) $(n=275)$. In details, among immunotherapy: 62\% received ipilimumab (anti-CTL A4), 25\% nivolumab/pebrolizumab (anti PD1), 13\% the two combined. Among BRAF therapy: 69\% received BRAF monotherapy (vemurafenib/dabrafenib), about 31\% received BRAF+MEK combination therapy (vemurafenib/dabrafenib + cobimetinib/trametinib). In the entire cohort the median overall melanoma-specific survival was 47 months (95\% CI: 40-53), the lowest median survival was detected by patients treated by chemotherapy (33 months, 95\% CI 27-38) as first option. Among immunotherapy the $M-s S$ globally was 50 months (95\% CI 43-57), it varied from 47 months (95\% CI 37-56) for ipilimumab (anti-CTL A4) to 70 months (95\% CI 39-101) for nivolumab/pebrolizumab (anti-PD 1). Target therapy globally produced $M-s S$ of 44 months (95\% CI 38-50), it varied from 40 months (95\% CI 34-45) for Vemurafenib/Dabrafenib single agent (anti-BRAF) to 55 months (95\% CI 49-61) for and the addition of Cobimetinid/Trametinib (anti-MEK).

Table 4. Results of the performance indicators on the quality of metastatic melanoma care Univariate Analysis

\begin{tabular}{|c|c|c|}
\hline \multirow[b]{2}{*}{ Indicator } & \multicolumn{2}{|c|}{$\begin{array}{l}\text { ADVANCED MELANOMA: } \\
\text { IIIB-IIIC (UNRESECTABLE), IV }\end{array}$} \\
\hline & $\begin{array}{c}\text { Observed } \\
\text { patients (n) }\end{array}$ & $(\%)$ \\
\hline Advanced melanoma patients & 966 & $(81.5)$ \\
\hline Patients excluded from the analysis for missing therapies & 179 & $(18.5)$ \\
\hline Patients eligible for analysis & 787 & (100) \\
\hline Patients with local therapy & 275 & (35) \\
\hline Patients with systemic therapy: chemotherapy & 275 & (35) \\
\hline Patients with systemic therapy: immunotherapy & 319 & $(41)$ \\
\hline Immunotherapy: ANTI-PD 1 (Nivolumab/Pebrolizumab) & 80 & $(25.1)$ \\
\hline Immunotherapy: ANTI-CTL A4 (Ipilimumab) & 198 & (62.1) \\
\hline
\end{tabular}


Immunotherapy: ANTI-PD 1 + ANTI-CTL A4

Patients with target therapy (anti-BRAF, BRAF + MEK)

Anti-BRAF: vemurafenib/dabrafenib

BRAF+MEK: cobimetinib/trametinib

Long-term outcomes

Melanoma-specific Survival (M-sS) overall

Melanoma-specific Survival (M-sS) of pts. with local therapy

Melanoma-specific Survival (M-sS) of pts. with chemotherapy

Melanoma-specific Survival (M-sS) of pts. with immunotherapy

M-sS Immunotherapy: ANTI-PD 1 (Nivolumab/Pebrolizumab)

$M-s S$ Immunotherapy: ANTI-CTL A4 (Ipilimumab)

$M-s S$ Immunotherapy: ANTI-PD 1+ANTI-CTL A4

Melanoma-specific Survival (M-sS) of pts. with target therapy

$M-s S$ Anti-BRAF: vemurafenib/dabrafenib

$M-s S$ BRAF+MEK: cobimetinib/trametinib

41

285

198

87

(12.8)

(36)

(69.5)

(30.5)

\begin{tabular}{cc} 
Events $^{2}$ (n) & Median M-s S, \\
DOD/DEAD & months (95\% CI) \\
$314 / 353$ & $47(41-53)$ \\
$132 / 147$ & $42(35-48)$ \\
$151 / 163$ & $33(27-38)$ \\
$126 / 137$ & $50(43-57)$ \\
$17 / 18$ & $70(39-101)$ \\
$94 / 104$ & $47(37-56)$ \\
$15 / 15$ & $58(26-90)$ \\
$129 / 147$ & $44(38-50)$ \\
$91 / 107$ & $40(34-45)$ \\
$38 / 40$ & $55(49-61)$ \\
\hline
\end{tabular}

${ }^{1}$ The number displayed corresponds to the number of hospitals. ${ }^{2}$ Event: number of deaths of the disease(DOD)/ number of deaths for all causes (DEAD).

Immunotherapy showed animproved survival compared with all other therapies $(p=0.001)$ (Fig. 2, A); for Ipilimumab and combined target therapy compared with all other therapies a slight significance were observed $(\mathrm{p}=0.05)$ (see Fig. $2 \mathrm{~B}$ ). The highest survival (70 months; 95\% CI 45-96) was reached by patients treated with Nivolumab/Pembrolizumab compared with combined target therapy and all other therapies $(p=0.001)$ (see Fig.2 $\mathrm{C}$ ); Immunotherapy across strata showed an improved survival for anti-PD1 and combined anti-PD $1+$ anti-CTL A4 compared with Ipilimumab and all other therapies $(\mathrm{p}<0.0001)$ (see Fig.2 D). The treatment-sequence did not show any significant difference (Immuno in $1^{\text {st }}$ and Target in $2^{\text {nd }}$ vs. Target in $1^{\text {st }}$ and Immuno in $2^{\text {nd }}$ line $)(p=0.5)$ (see Fig.2 E). A significant difference was observed between BRAF vs. BRAF with the addition of Cobimetinid/Trametinib (anti-MEK) ( $\mathrm{p}=0.03$ ) (see Fig. 2F). 
A Overall Survival (OS) Immunotherapy

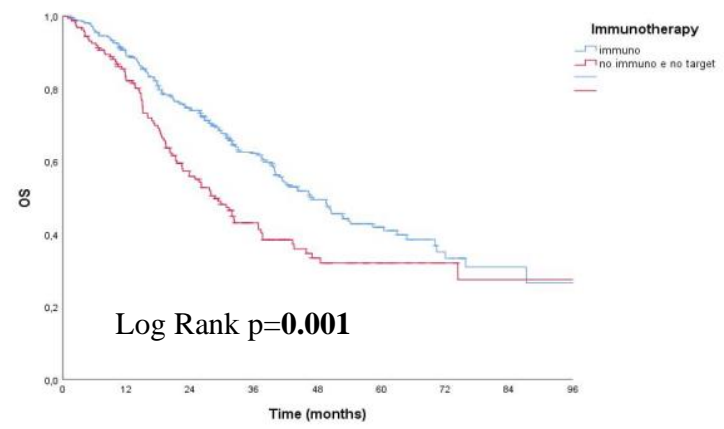

C OS Immuno: ANTI-PD 1

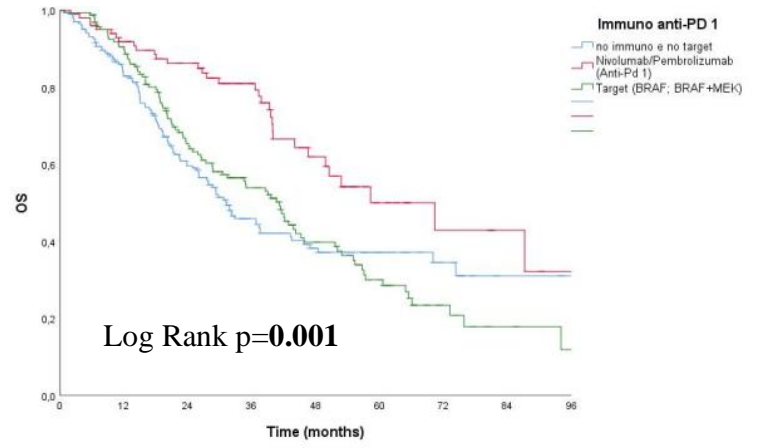

B: OS Immunotherapy: ANTI-CTL A4

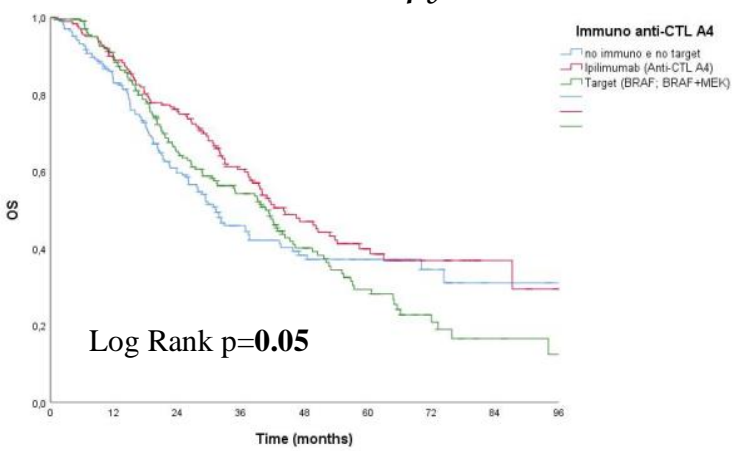

$D$ OS Immuno: ANTI-PD 1; ANTI-CTL A4; ANTI-PD 1+ANTI-CTL A4

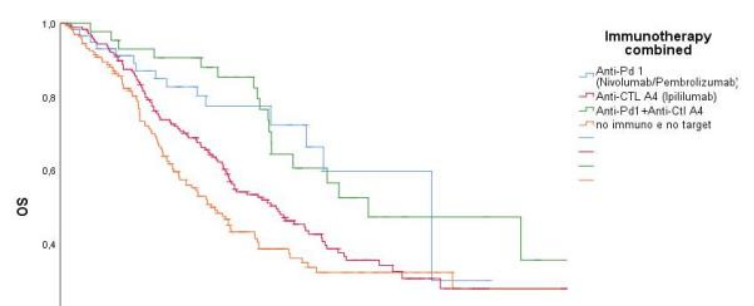

Log Rank p $<\mathbf{0 . 0 0 0 1}$

E OSTreatment Sequence:Immuno $1^{\text {st }}, 2^{\text {nd }}$; Target $1^{\text {st }}, 2^{\text {nd; }}$ Target $1^{\text {st }} \&$ Immuno $2^{\text {nd }}$ F $\quad$ OS BRAF vs. BRAF+MEK:
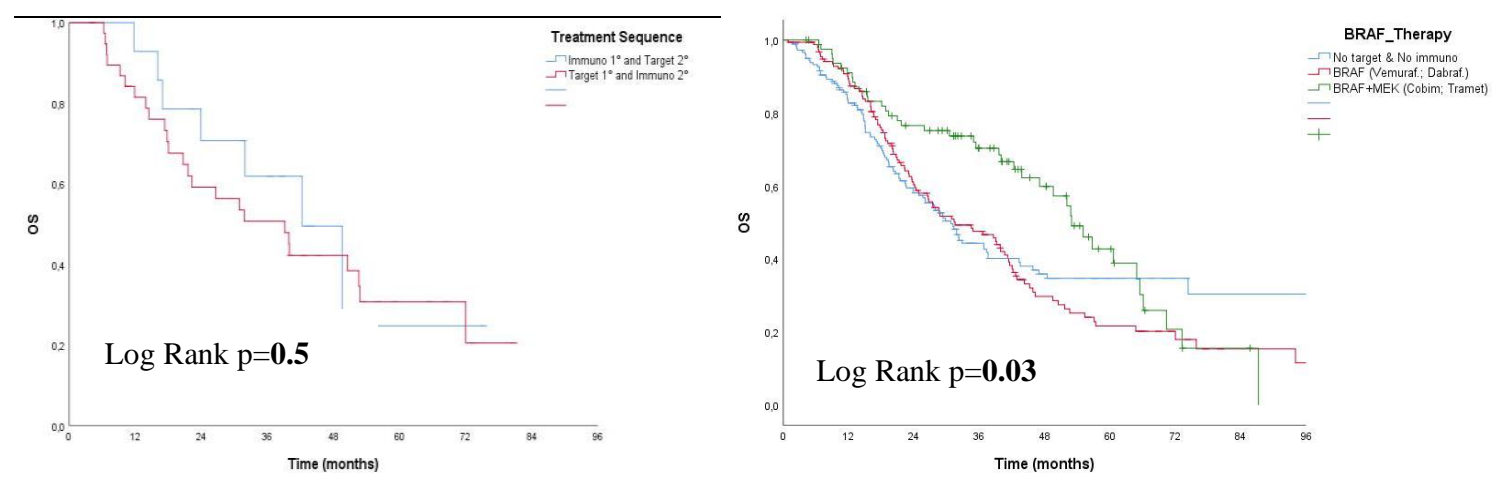

Figure 2. Overall Survival (OS) in patients with IIIB-IIIC (UNRESECTABLE), IV by Therapy (A, B, C, D, E, F)

Multivariate Cox model hazard ratios were reported in Table 5: a significant increased risk of death was observed for abnormal LDH compared to normal (HR=1.94 95\% CI 1.23-3.06); among the Target therapy a significant protective effect was observed for target therapy with the addition of Cobimetinid/Trametinib (BRAF+MEK) (HR=0.63 95\% CI 0.42-0.94). All immunotherapy categories were significantly associated with a reduction of death: anti-PD1 HR=0.25 (95\% CI 0.15-0.43), anti-CTL A4 HR=0.47 (95\% CI 0.33-0.67) and combined anti-PD1+ anti-CTL A4 HR=0.26 (95\% 
CI 0.15-0.47), respectively. The treatment-sequence was not associated to the risk of death $(\mathrm{p}=0.3)$.

Table 5.Univariate and multivariate Cox regression models for death

\begin{tabular}{|c|c|c|c|}
\hline \multirow[t]{2}{*}{ Parameter / Category } & \multicolumn{3}{|c|}{ Adjusted Multivariate Analysis ${ }^{\ddagger}$} \\
\hline & HR & $95 \% \mathrm{CI}$ & $\mathbf{p}$ \\
\hline \multicolumn{4}{|l|}{ LDH } \\
\hline Normal & $1.0^{+}$ & & \\
\hline Abnormal & 1.95 & $1.24-3.01$ & 0.004 \\
\hline Unknown & 0.97 & $0.95-1.53$ & 0.09 \\
\hline \multicolumn{4}{|l|}{ Target therapy } \\
\hline No Target and No Immuno therapy & $1.0^{+}$ & & \\
\hline$B R A F$ & 1.14 & $0.85-1.53$ & 0.4 \\
\hline$B R A F+M E K$ & 0.623 & $0.42-0.94$ & 0.02 \\
\hline \multicolumn{4}{|l|}{ Immunotherapy } \\
\hline No Immuno and No Target therapy & $1.0^{+}$ & & \\
\hline ANTI-PD 1 (Nivolumab/Pebrolizumab) & 0.25 & $0.147-0.43$ & $<0.0001$ \\
\hline ANTI-CTL A4 (Ipilimumab) & 0.47 & $0.33-0.67$ & $<0.0001$ \\
\hline ANTI-PD 1+ ANTI-CTL A4 & 0.26 & $0.15-0.47$ & $<0.0001$ \\
\hline \multicolumn{4}{|l|}{ Treatment Sequence } \\
\hline Immuno $1^{\text {st }}$ and Target $2^{\text {nd }}$ & $1.0^{+}$ & & \\
\hline Target $1^{\text {st }}$ and Immuno $2^{\text {nd }}$ & 1.64 & $0.65-4.12$ & 0.3 \\
\hline
\end{tabular}

${ }^{+}$Reference category; $\ddagger$ MultivariateCox model adjusted for gender (male, female); age ( $\left.\leq 60,>60\right)$; geographical area (North, Central-South); Year BRAF executed ( $\leq 2013,>2013)$; N. of therapies $(1,2$, 23); Other therapies: Chemotherapy; Local and systemic therapy whenever. 


\section{Discussion}

In this study, we examined data of advanced melanoma in the Italian Clinical National Melanoma Registry (CNMR). CNMR doesnot have the typical aim of cancer registries to estimate incidence data, but as a clinical registry may collect data from the real world experience which is different from that coming from clinical studies which included selected patients. [22-23]. Indeed, much of the existing research on advanced melanoma patients has been conducted in clinical trials settings among patients who meet stringent inclusion and exclusion criteria.

The analysis of the 787 patients from the advanced cohort showed some interesting results. As first, looking at the advanced patients' characteristics, a good percentage of them come from the initial stages more than from the high risk conditions. Indeed, 50\% of advanced melanoma had an initial diagnosis of early stage that then developed into advanced one.

Interestingly, the BRAF mutational status wasnot evaluated in all patients; indeed, the BRAF status has been documented in as much as $76 \%$ of these patients.An important consideration is that the CNMR collected data from December 2011 and the most important drug in the field of melanoma, like BRAF inhibitors, anti-CTLA4, anti-PD1 were approved in the following years. Specifically ipilimumab was the first treatment to be approved, on February 2013, by AIFA. Vemurafenib and dabrafenib received approval on May 2013 and on October 2014 respectively as monotherapy, and on September 2016 and on January 2017 in combination with cobimetinib and trametinib respectively. Pembrolizumab was approved on May 2016 while nivolumab on 24 March 2016(24). Moreover, the possibility to ask for the BRAF mutational status was probably related only to the centers which were participating to clinical studies or expanded access programs with such drugs.

Another important analysis is that related to the OS. With all the limitation of the analysis due mainly to the time of collection of such data (before the largely use of anti-PD-1 and BRAF/MEK inhibitors, and the small number of patients considered), there are still some interesting findings. It is evident that the new therapies available had an important impact on the survival of these patients. Indeed, patients who practiced immunotherapy or target 
therapy performed better in terms of median survival than those who practiced local therapy and / or chemotherapy, considered for a long time the only standard of treatment for metastatic melanoma. The addition of the MEK inhibitor to the BRAF inhibitor significantly improved patient OS.

It seems that the greater advantage in terms of OS is in those patients who have performed immunotherapy lines, even compared to those who have performed target therapies. This finding could be explained by the fact that many patients received BRAF inhibitor therapy as single agent $(69,5 \%)$, and only a minority had benefit from the addition of the MEK inhibitor. Indeed, we learned that disease progression during therapy with the BRAF inhibitor alone was often rapid and unresponsive to subsequent treatments [25]; with the addition of MEK inhibitors, the fast progression from target therapy was reduced.

The data on the combination nivolumab + ipilimumab also appears intriguing, especially in terms of long survival; however, the low number of patients does not allow us to give definitive conclusions.

The correlation between survival and the $\mathrm{LDH}$ value is also consistent with the literature data. Analyzing the LDH values, there is an increased risk of death for patients with high $\mathrm{LDH}$, compared to those with normal LDH, especially in the group of patients who received immunotherapy $(\mathrm{HR}=2.45, \mathrm{p}=0.01)$

We found that immunotherapy allows better results in terms of overall survival in patients with advanced melanoma, however in our analysis there is no statistically significant benefit of the treatment-sequence variable (Immuno in $1^{\text {st }}$ and Target in $2^{\text {nd }}$ vs. Target in $1^{\text {st }}$ and Immuno in $2^{\text {nd }}$ line). In consideration of the retrospective analysis, the small number of patients who started with anti-PD-1, and the lack of patients who received the dual MAPK blockade, definitive conclusions cannot be made.

At the moment several combination studies of target and immunotherapies as well as protocols to establish the best sequential therapy areongoing. [26]. Our study has several limitations. In fact, most patients received chemotherapy as a first systemic treatment for 
advanced disease, because more effective drugs such as BRAF/MEK inhibitors, anti-CTLA4 and anti-PD1 inhibitors were approved subsequently in different years. In addition, many centers did not test all patients for BRAF, especially at the beginning.

\section{Materials and Methods}

\subsection{Patients and data collection}

CNMR is the first clinical registry established in Italy in 2010. It collects data from a wide network of melanoma centers throughout the country with the aim to carry out clinical and therapeutic evaluations investigating geographical and policy differences and instruments for planning specific health interventions in different populations and areas, in order to optimize the clinical management and survival of CMM patients. CNMR collects data of patients with a histologically confirmed diagnosis of primary CMM treated in 38 Italian institutions (hospitals, research institutes, ecc.) participating in the network, as previously described [21]. For the purposes of the present study, data of consecutive patients enrolled from January 2011 to December 2018 were considered (CNMR established in 2010 but the first year was spent for administrative approvemnet and ethical commette in each centres).

A diagram of the CNMR's organizational structure can be found in Figure 1.

\section{Organizational structure of Clinical National Melanoma Registry (CNMR)}

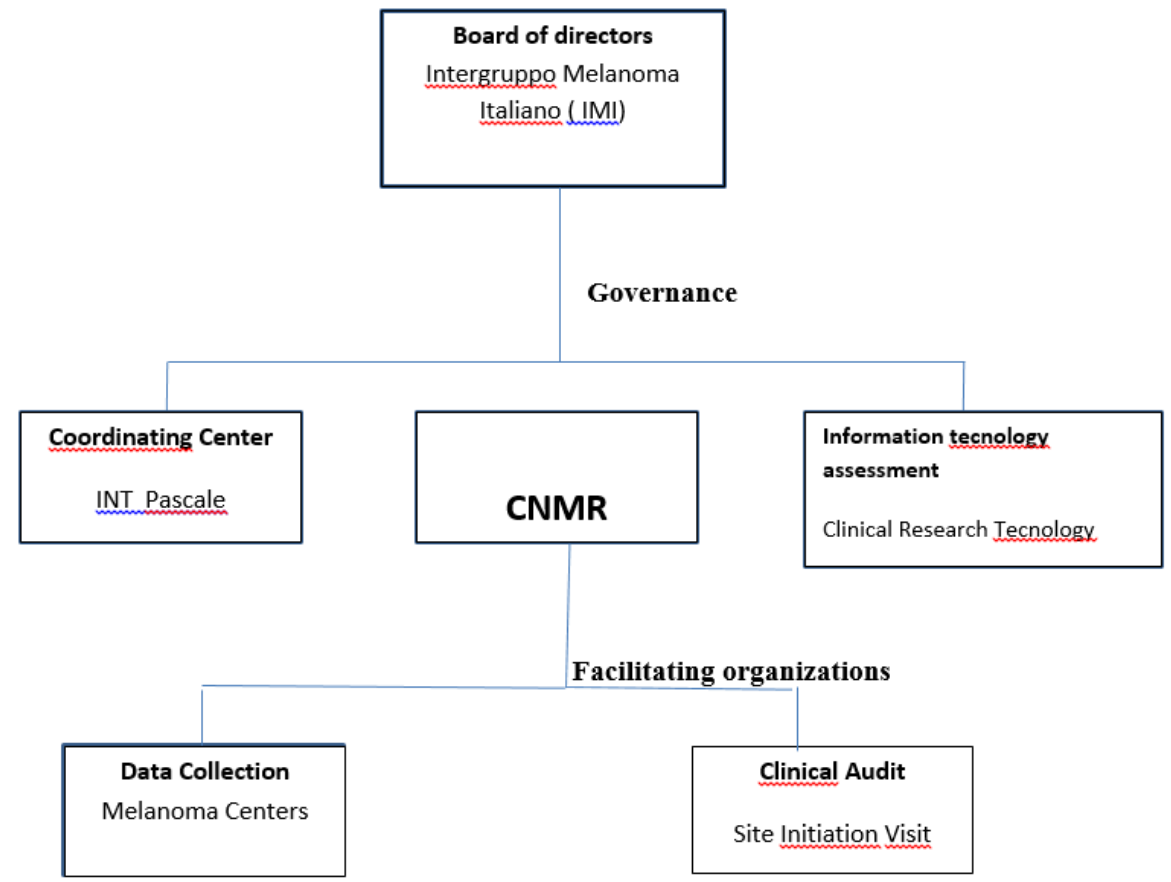


Figure 1. Integrated management of CNMR. Two main complementary approaches are considered-Governance and Faciliting organization.

Data were collected via an electronic Case Report Form (eCRF), which was developed by the Clinical Research Technology S.r.l. group (Salerno, Italy) on its clinical platform 'eClinical'. 'eClinical' assigned an identification (ID) number to all the patients screened. The quality of the electronic data was verified through onsite clinical visits, undertaken periodically during the study. The eCRF was designed to collect information on sociodemographic, clinical, pathological and treatment variables. The type of therapy was registered in all cases: local therapy (radiotherapy and electro-chemotherapy), systemic chemotherapy (platinum salts, dacarbazine, fotemustine), targeted therapy (anti-BRAF: vemurafenib/dabrafenib; BRAF+MEK: cobimetinib/trametinib), and immunotherapy (anti-CTLA4: ipilimumab, anti-PD1: nivolumab/pembrolizumab; and anti-CTLA4 + anti-PD1). Further information regarding the date of diagnosis, the duration of therapy, the date of the last follow-up, and the clinical status of the patients were also registered.

\subsection{Statistical analysis}

Descriptive statistics for the categorical data were reported. Pearson's Chi-squared was used to compare categorical variables. Eligible patients for the survival analysis had histologically confirmed, unresectable stage III or stage IV metastatic melanoma (stage IIIB-IV) with an Eastern Cooperative Oncology Group (ECOG) performance status 0 or 1, and known BRAF mutation status. All patients were followed until 31 December 2018 or until the date of last visit, or death, whichever came first.

Melanoma-specific survival (M-s S) was calculated from the date of initial adjuvant treatment to death for the disease and Overall survival (OS) until date of death from any cause. Patients who did not die were censored for OS on the last visit date available in the database. When the date of diagnosis was antecedent the beginning of the Melanoma Registry or the initial diagnosis was an early melanoma we considered the M-s Survival from the date of initial adjuvant treatment. 
Kaplan-Meier curves and medians of OS and 95\% CI are presented overall and by immunotherapy and target treatments. The Log-rank test compared curves by treatments (immunotherapy: anti-CTLA4, anti-PD1 vs. no immunotherapy and no target therapy; BRAF: anti-BRAF, BRAF+MEK vs. no immunotherapy and no target therapy). Univariate and multivariable Cox regression models were used to estimate the hazard ratios adjusting for confounders and other prognostic factors.

All statistical tests were two-sided. P-values $<0.05$ were considered significant. Statistical analyses were performed using statistical software SAS (version 9.02 for Windows), and Statistical Package for Social Science (SPSS) version 25 (SPSS inc., Chicago IL, USA).

\section{Conclusions}

Finally, this study shows that immunotherapy improves survival in advanced melanoma in a real-world population. The CNMR represents a set of data useful not only to plan the appropriate prevention measures but to better understand the effectiveness of anti-cancer treatments in a large unselected population from a real world experience. Furthermore, qualified data is essential and it is important that this information is constantly updated in order to maintain high levels of evidence.

The nivolumab/pembrolizumab and the combination of ipilimumab can be considered the best therapy to improve survival in a real-world-population. The CNMR can complement clinical registries with the intent of improving cancer management and standardizing cancer treatment.

Author Contributions Conceptualization, A.C., G.P., A.T., and P.A.A.; methodology, A.C., V.V., C.C., G.G., M.B., G.M., F.T., S.G., G.P., P.A.A; software, A.C., and S.G.; validation, A.C., M.T.C., A.V., P.D.F., P.Q., F.S., V.V., C.C., G.G., S.R., G.M., E.C., M.B., F.G., S.G, A.T., G.P., and P.A.A.; formal analysis, A.C., V.V., S.G.; investigation, M.T.C., E.G., A.V., P.D.F., P.Q., F.S., V.V., C.C., G.T., E.P., G.G., E.N., P.Q., S.R., M.G., D.M., S.B., M.G., V.D.G., M.O., F.G., G.C., A.G., D.M., L.A., N.C., T.M., G.M., F.T., E.C., M.B., S.G, C.R.R, A.T., and P.A.A.; resources A.V., M.B., C.R.R., A.T., G.P., F.S., E.P., V.V., C.C., G.T., S.R., M.B., G.M., S.G, A.T., G.P., and P.A.A.; writing-review and editing A.C., V.V., G.T., G.G., G.M., E.C., M.G., M.B., L.A., S.G, A.T., G.P., and P.A.A.; visualization, A.C., P.Q., F.S., E.P., V.V.,D.M., F.G., S.B., M.G., F.T., L.A., T.F., S.G., G.P., P.A.A; supervision, A.C., E.C., S.G., G.P., P.A.A; project administration A.V., P.Q., M.T.C., G.M., M.B., S.G., G.P., P.A.A; funding acquisition, C.R.R., A.T., P.A.A. All authors have read and agreed to the published version of the manuscript."

\section{Ethics approval}


This study was approved by the ethics committee of the National Cancer Institute "Fondazione Giovanni Pascale" in Naples, with the protocol number 537/10 registration date October 27, 2010.

Funding: This research was funded by grants received from Bristol Myers Squibb (New York, NY, USA), GlaxoSmithKline (Brentford, UK) and Pierre Fabre Pharma.

Acknowledgments: We would like to express our special thanks to Dr. Maurizio Montella († May 2, 2019), for his long-term contribution to the study and for his ideas and support of this current manuscript.

The authors would like to thank the Intergruppo Melanoma Italiano (IMI) and the Clinical Research Technology (CRT).

Conflicts of Interest: Paolo A. Ascierto has/had a consultant/advisory role for Bristol Myers Squibb, Roche-Genentech, Merck Sharp \& Dohme, Novartis, Array, Merck Serono, Pierre-Fabre, Incyte, Medimmune, AstraZeneca, Syndax, Sun Pharma, Sanofi, Idera, Ultimovacs, Sandoz, Immunocore, 4SC, Alkermes, Italfarmaco, Nektar, Boehringer-Ingelheim, Eisai, Regeneron, Daiichi Sankyo, Oncosec, Pfizer. He also received research funding from Bristol Myers Squibb, Roche-Genentech, Array and travel support from MSD.

\section{References}

1. Ferlay J, Shin HR, Bray F, Forman D, Mathers C and Parkin DM: Estimates of worldwide burden of cancer in 2008: GLOBOCAN 2008. Int J Cancer 127: 2893-2917, 2010.

2. Hollestein LM, de Vries E and Nijsten T: Trends of cutaneous squamous cell carcinoma in the Netherlands: Increased incidence rates, but stable relative survival and mortality 1989-2008. Eur J Cancer 48: 2046-2053, 2012.

3. de Vries E, Bray FI, Coebergh JW and Parkin DM: Changing epidemiology of malignant cutaneous melanoma in Europe 1953-1997: Rising trends in incidence and mortality but recent stabilizations in western Europe and decreases in Scandinavia. Int J Cancer 107: 119-126, 2003.

4. Garbe C and Leiter U: Melanoma epidemiology and treands. Clin Dermatol 27:3-9,2009 Crocetti E, et al. Survival of patients with skin melanoma in Europe increases further: Results of the EUROCARE-5 study. Eur J Cancer. 2015 Oct;51(15):2179-2190.

5. Cossu A, et al. Epidemiology and genetic susceptibility of malignant melanoma in North Sardinia, Italy. Eur J Cancer Prev. 2017 May;26(3):263-267.

6. AIOM and AIRTum Working Group and Working Group. Il numero del cancro in Italia 2019.

7. Sini MC, Doneddu V, Paliogiannis P, Casula M, Colombino M, Manca A, Botti G, Ascierto PA, Lissia A, Cossu A, Palmieri G. Geneticalterations inmaincandidategenesduring melanoma progression.Oncotarget. 2018 Jan 3;9(9):8531-8541. doi: 10.18632/oncotarget.23989. 
8. Palmieri G, Ombra M, Colombino M, Casula M, Sini M, Manca A, Paliogiannis P, Ascierto PA, Cossu A Multiple Molecular Pathways in Melanomagenesis: Characterization of Therapeutic Targets.. Front Oncol. 2015 Aug 10;5:183.

9. Chapman PB, et al. Improved survival with vemurafenib in melanoma with BRAF V600E mutation. N Engl J Med. 2011 Jun 30;364(26):2507-16.

10. Hauschild A, et al. Dabrafenib in BRAF-mutated metastatic melanoma: a multicentre, open-label, phase 3 randomised controlled trial. Lancet. 2012 Jul 28;380(9839):358-65.

11. Long GV,et al. Dabrafenib plus trametinib versus dabrafenib monotherapy in patients with metastatic BRAFV600E/K-mutant melanoma: long-term survival and safety analysis of a phase 3 study. Ann Oncol. 2017 Jul 1;28(7):1631-1639.

12. Robert C, Grob JJ, Stroyakovskiy D, Karaszewska B, Hauschild A, Levchenko E, Chiarion Sileni V, Schachter J, Garbe C, Bondarenko I, Gogas H, Mandalá M, Haanen JBAG, Lebbé C, Mackiewicz A, Rutkowski P, Nathan PD, Ribas A, Davies MA, Flaherty KT, Burgess P, Tan M, Gasal E, Voi M, Schadendorf D, Long GV. Five-Year Outcomes with Dabrafenib plus Trametinib in Metastatic Melanoma.N Engl J Med. 2019 Aug 15;381(7):626-636.

13. Larkin J, et al. Combined vemurafenib and cobimetinib in BRAF-mutated melanoma. N Engl J Med. 2014 Nov 13;371(20):1867-76.

14. Topalian SL et al. Safety, activity, and immune correlates of anti-PD-1 antibody in cancer. N Engl J Med 366(26):2443-2454.

15. Hodi FS et al. Improved survival with ipilimumab in patients with metastatic melanoma N Engl J Med. 2010 Aug 19;363(8):711-23.

16. Schadendorf D, Hodi FS, Robert C, Weber JS, Margolin K, Hamid O, Patt D, Chen TT, Berman DM, Wolchok JD. Pooled Analysis of Long-Term Survival Data From Phase II and Phase III Trials of Ipilimumab in Unresectable or Metastatic Melanoma.J Clin Oncol. 2015 Jun 10;33(17):1889-94. doi: 10.1200/JCO.2014.56.2736. Epub 2015 Feb 9.

17. Ascierto PA, Del Vecchio M, Robert C, Mackiewicz A, Chiarion-Sileni V, Arance A, Lebbé C, Bastholt L, Hamid O, Rutkowski P, McNeil C, Garbe C, Loquai C, Dreno B, Thomas L, Grob JJ, Liszkay G, Nyakas M, Gutzmer R, Pikiel J, Grange F, Hoeller C, Ferraresi V, Smylie M, Schadendorf D, Mortier L, Svane IM, Hennicken D, Qureshi A, Maio M. Ipilimumab 10 mg/kg 
versus ipilimumab $3 \mathrm{mg} / \mathrm{kg}$ in patients with unresectable or metastatic melanoma: a randomised, double-blind, multicentre, phase 3 trial.Lancet Oncol. 2017 May;18(5):611-622. doi: 10.1016/S1470-2045(17)30231-0. Epub 2017 Mar 27.PMID: 28359784 Clinical Trial.

18. Schachter J, et al. Pembrolizumab versus ipilimumab for advanced melanoma: final overall survival results of a multicentre, randomised, open-label phase 3 study (KEYNOTE-006). Lancet. 2017 Oct 21;390(10105):1853-1862.

19. Robert C, et al. Nivolumab in previously untreated melanoma without BRAF mutation. N Engl J Med. 2015 Jan 22;372(4):320-30.

20. Larkin J, Chiarion-Sileni V, Gonzalez R, Grob JJ, Rutkowski P, Lao CD, Cowey CL, Schadendorf D, Wagstaff J, Dummer R, Ferrucci PF, Smylie M, Hogg D, Hill A, Márquez-Rodas I, Haanen J, Guidoboni M, Maio M, Schöffski P, Carlino MS, Lebbé C, McArthur G, Ascierto PA, Daniels GA, Long GV, Bastholt L, Rizzo JI, Balogh A, Moshyk A, Hodi FS, Wolchok JD. Five-Year Survival with Combined Nivolumab and Ipilimumab in Advanced Melanoma.N Engl J Med. 2019 Oct 17;381(16):1535-1546. doi: 10.1056/NEJMoa1910836. Epub 2019 Sep 28.

21. Gandini S, Montella M, Ayala F, Benedetto L, Rossi CR, Vecchiato A, Corradin MT, DE Giorgi V, Queirolo P, Zannetti G, Giudice G, Borroni G, Forcignanò R, Peris K, Tosti G, Testori A, Trevisan G, Spagnolo F, Ascierto PA, CLINICAL NATIONAL MELANOMA REGISTRY GROUP Sun exposure and melanoma prognostic factors. Oncol Lett. 2016 Apr;11(4):2706-2714.

22. Jochems A, Schouwenburg MG, Leeneman B, Franken MG, van den Eertwegh AJ, Haanen JB, Gelderblom H, Uyl-de Groot CA, Aarts MJ, van den Berkmortel FW, Blokx WA, Cardous-Ubbink MC, Groenewegen G, de Groot JW, Hospers GA, Kapiteijn E, Koornstra RH, Kruit WH, Louwman MW, Piersma D, van Rijn RS, Ten Tije AJ, Vreugdenhil G, Wouters MW, van der Hoeven JJ. Dutch Melanoma Treatment Registry: Quality assurance in the care of patients with metastatic melanoma in the Netherlands. Eur J Cancer. 2017 Feb;72:156-165. doi: 10.1016/j.ejca.2016.11.021. Epub 2016 Dec 25.

23. Cowey CL, Liu FX, Boyd M, Aguilar KM, Krepler C.Real-world treatment patterns and clinical outcomes among patients with advanced melanoma: A retrospective, community oncology-based cohort study (A STROBE-compliant article). Medicine (Baltimore). 2019 Jul;98(28):e16328. 
24. AIFA (Regime di rimborsabilita' e prezzo di vendita del medicinale nivolumab) - autorizzata con procedura centralizzata europea dalla Commissione europea. (Gazzetta Ufficiale della Repubblica Italia n.70 24-03-2016.

25. Ascierto PA, Simeone E, Grimaldi AM, Curvietto M, Esposito E, Palmieri G, Mozzillo N. Do BRAF inhibitors select for populations with different disease progression kinetics? J Transl Med. 2013 Mar 8;11:61. doi: 10.1186/1479-5876-11-61.

26. Pavlick AC, Fecher L, Ascierto PA, Sullivan RJ. Frontline Therapy for BRAF-Mutated Metastatic Melanoma: How Do You Choose, and Is There One Correct Answer? Am Soc Clin Oncol Educ Book. 2019 Jan;39:564-571. doi: 10.1200/EDBK_243071. Epub 2019 May 17. 